will not occur on an ad hoc basis, but will function in an institutionalised manner.

- Adjustment of the legal framework: many countries have constitutions which do not contain any provisions on parliaments and sending troops abroad in peace support operations. Many constitutions were drafted in the $19^{\text {th }}$ or beginning of the $20^{\text {th }}$ century, in which period peace support operations played a limited role, if any. These constitutions only deal with parliamentary consent to the declaration of war. However, nowadays we are faced with undeclared wars only. Therefore some parliaments, for example the Netherlands' parliament, has amended its constitution and strengthened its position in the issue of sending troops abroad on peace support operations.

- Effective rules of procedure: another obstacle is the confidentiality and secrecy which decision-making on peace support operations sometimes requires. Various parliaments have developed simple but practical rules of procedure in order to deal with classified information, e.g. vetting/clearance procedures of defence committee members, convening behind closed doors, making strict distinction between public reports and classified reports or procedures for declassifying documents after the PSOs are finished - which makes post-accountability possible.

- Accountability: concerning the willingness of parliaments to keep the government accountable, the potential negative influence of party discipline on the freedom of MPs to raise critical questions or to vote against the gov- ernment is difficult to overcome. Government parties often have the majority in parliament and can block any initiative coming from the opposition. In this respect, in some countries, e.g. Hungary, approval of PSOs requires a majority of two-thirds in parliament which implies that government parties have to cooperate with opposition parties. In other countries, the parliamentary rules of procedure require that the chair of the defence committee is given to the opposition.

To conclude, these examples, and there are many others, show that the double democratic deficit can be transformed into a democratic surplus. However, strengthening parliamentary oversight can only be done with a particular role for parliament in mind. Parliament can fulfil a strong role and become an equal partner to the government. Parliaments can also fulfil a weak role which diminishes their role to an institution which rubberstamps government decisions. At the end we have to answer the question how strong parliament should be and where to draw the 'dividing' line of competences between government and parliament. From the point of view of a 'government of the people, by the people and for the people', the bottom line is that parliamentary accountability is indispensable. It is difficult to imagine that such an important issue as sending troops abroad in PSOs is excluded from democratic decisionmaking processes, since parliaments are the most important provider of democratic legitimacy.

\title{
Die Abschaffung der Wehrpflicht - kein Schaden für die Innere Führung
}

\author{
Jürgen Groß*
}

\begin{abstract}
There are no longer any valid reasons to retain compulsory military service in Germany. After years of public debate during which every possible argument has been used in every possible way, a political decision to change the form of Germany's armed forces is long overdue. This would not hinder the necessary expansion of democratisation in the German military (»Innere Führung«) - quite the contrary.
\end{abstract}

Keywords: Conscription vs. voluntary military service, Democratisation of the armed forces.

I.

A lle sicherheitspolitischen und militärischen Argumente sprechen längst für Freiwilligenstreitkräfte, denn was nützen uns Wehrpflichtige, die mit großem Aufwand eingezogen und ausgebildet werden, aber

\footnotetext{
* Dr. Jürgen Groß, Oberstleutnant i.G., Institut für Friedensforschung und Sicherheitspolitik an der Universität Hamburg (IFSH).
}

dann für die entscheidenden Aufgaben, nämlich Auslandseinsätze, gar nicht zur Verfügung stehen? Wer will es verantworten, unzureichend ausgebildete Grundwehrdienstleistende in höchst riskante Einsätze zu schicken? Unsere wichtigsten Verbündeten sind nicht ohne Grund denn auch längst zu Freiwilligenstreitkräften übergegangen - haben sie etwa alle Unrecht? Und mögen sie dabei in mancherlei Hinsicht nicht nur gute Erfahrungen gemacht haben - man könnte ja daraus lernen und es selbst besser machen. 
Im Übrigen können diese (oft aufgebauschten) Detailprobleme so gravierend auch wieder nicht sein - jedenfalls denkt keiner dieser Staaten ernsthaft daran, die Wehrpflicht erneut einzuführen.

Auch unter dem Kostenaspekt kommt man zum selben Ergebnis, denn die Wehrpflicht ist teurer als man denkt: Selbstverständlich sind die budgetären Kosten für einen einzelnen Grundwehrdienstleistenden niedriger als die für einen Zeitsoldaten; wenn man jedoch alle volkswirtschaftlichen Kosten miteinbezieht (Wehrpflichtige zahlen keine Steuern und Sozialversicherungsbeiträge, erhalten Unterhaltsleistungen und vielfältige Vergünstigungen durch die Kommunen etc.), reduziert sich zum einen diese Differenz beträchtlich. Zweitens bindet die Wehrpflicht durch ihren permanenten Ausbildungs- und Verwaltungsaufwand in erheblichem Umfang selbst Personal und Ressourcen. So gesehen könnte man über die Wehrpflicht nicht einmal sagen: Nützt sie auch nichts, so schadet sie doch nur wenig. Und drittens interessieren in diesem Zusammenhang nur die insgesamt in den Streitkräften anfallenden Personalkosten kein seriöses Modell für Freiwilligenstreitkräfte geht von einem Personalumfang von 285.000 Soldatinnen und Soldaten, wie er bis vor kurzem für die Bundeswehr vorgesehen war, aus. Und auch der nunmehr vorgesehene Personalumfang von 250.000 Soldatinnen und Soldaten schöpft noch längst nicht alle möglichen Einsparpotenziale aus. Die Kommission »Europäische Sicherheit und Zukunft der Bundeswehr « am Institut für Friedensforschung und Sicherheitspolitik an der Universität Hamburg (IFSH) etwa hält in ihrem Bundeswehrmodell »200F« einen Personalumfang von maximal 200.000 für völlig ausreichend. ${ }^{1}$

Auch das militärische Argument, die Bundeswehr gewinne derzeit rund die Hälfte ihres Nachwuchses an Offizieren und Unteroffizieren aus den Reihen ihrer Wehrpflichtigen, kann nicht überzeugen. Denn ist die Nachwuchslage einer Armee nicht das Resultat einer Summe von Faktoren, die alle mit der Einschätzung der Attraktivität des Arbeitgebers Bundeswehr zu tun haben (auch im Vergleich zur zivilen Konkurrenz) - also eine Gleichung mit vielen Unbekannten, in die auch potenzielle Negativfaktoren, wie beispielsweise die mit hoher Glaubwürdigkeit ihrem sozialen Umfeld übermittelten Erlebnisberichte frustrierter Grundwehrdienstleistender, mit einzubeziehen wären? (Und dass es mit der Attraktivität der deutschen Streitkräfte nicht immer zum Besten bestellt ist, wird jedes Jahr aufs Neue nicht zuletzt durch den Bericht des Wehrbeauftragten des Deutschen Bundestags deutlich belegt.) Es ist jedenfalls nicht recht einzusehen, dass ausgerechnet der Umweg über einen Zwangsdienst der Bundeswehr den besseren Nachwuchs liefern sollte als etwa die Installierung eines attraktiv ausgestalteten Anreizsystems für die freiwillige Übernahme von öffentlichen Aufgaben, darunter auch beim Militär. ${ }^{2}$

1 Groß, Jürgen, Bundeswehr-Modell »200F «, in: Vierteljahresschrift für Sicherheit und Frieden (S+F) 4/2001, S. 176-179.

2 Vgl. dazu Meyer, Berthold, Der Ausweg aus dem Dilemma von Wehrpflicht und Wehrgerechtigkeit: freiwillige Dienste und Berufsfreiheit für alle, in: Groß, Jürgen (Hrsg.), Europäische Sicherheit und Zukunft der Bundeswehr. Analysen und Empfehlungen der Kommission am IFSH, Baden-Baden 2004, S. 53-62.
Mit anderen Worten: Es gibt etliche Stellen, an denen man ansetzen kann (ja sogar muss), um die Nachwuchslage weiter zu verbessern - das starre Beharren auf einer antiquierten Wehrform allein genügt sicher nicht. Doch selbst wenn es genügen würde: Es ist völlig unverhältnismäßig, Jahr für Jahr 100.000 Mann einzuziehen, nur um einige tausend Freiwillige zu bekommen.

\section{II.}

Das am häufigsten benutzte - und wohl auch das gewichtigste - Argument der Wehrpflichtbefürworter liegt aber auf der gesellschaftspolitischen Ebene, weshalb darauf etwas näher eingegangen werden soll. Doch ist es - bei näherem Hinsehen - ebenso unhaltbar. Denn zu einer Überschätzung der gesellschaftspolitischen Integrationsleistung der Wehrpflichtigen besteht absolut kein Anlass: Die allgemeine Wehrpflicht ist in der Bundesrepublik seit vielen Jahren kaum mehr als eine Fiktion. Die heutigen deutschen Streitkräfte umfassen längst nicht mehr alle männlichen Bürger, unabhängig von Herkunft, Beruf und Bildung, und somit auch nicht mehr das gesamte Spektrum der vorhandenen Einstellungen. Die soziale Kontrolle ist damit keineswegs so wirkungsvoll wie vielfach behauptet. Die Integrationskraft der in der Bundesrepublik praktizierten Wehrpflicht darf also nicht überbewertet werden.

Zudem kann davon ausgegangen werden, dass, alles in allem, die Einstellungen der jüngeren Freiwilligen mit kurzer Verpflichtungszeit von den Einstellungen der derzeitigen Grundwehrdienstleistenden oder »Freiwillig Zusätzlichen Wehrdienst Leistenden « wohl gar nicht so weit entfernt liegen. Eine drastische Reduzierung des Berufssoldatenanteils sowie eine auf ein vertretbares Mindestmaß begrenzte Dienstzeit der übrigen Freiwilligen erscheinen überdies als wirkungsvolle und wohl hinreichende Gegenmaßnahmen zur Verhinderung unerwünschter Entfremdungsprozesse und sind daher auch die Kennzeichen seriöser alternativer Strukturmodelle, wie etwa des erwähnten Bundeswehrmodells $2200 \mathrm{~F} \ll$.

Doch selbst wenn man einer Wehrpflichtarmee unerreichbare Integrationsleistungen konzedieren wollte - was wie gesagt nicht der Fall ist -, so wäre zu berücksichtigen, dass in dieser Hinsicht neben der Wehrform zahlreiche weitere Variablen relevant sind, die die unterstellten Defizite von Freiwilligenstreitkräften gegebenenfalls mehr als kompensieren könnten. Es gibt weit effizientere Wege, Streitkräfte in der Demokratie zu verankern und der Kontrolle durch eine pluralistische Gesellschaft zu unterwerfen, als das Festhalten an einer aus vielerlei Gründen unzeitgemäßen Wehrform. Auch dazu haben die oben genannte Kommission sowie ein in Absprache mit dem Wehrbeauftragten des Deutschen Bundestages eingerichteter Arbeitskreis »Weiterentwicklung der 
Inneren Führung « weitreichende und zugleich detaillierte Vorschläge erarbeitet. ${ }^{3}$

Im Übrigen: Gerade dort, wo demokratische Kontrolle am notwendigsten ist, auf den Führungsebenen nämlich, waren Wehrpflichtige aus einleuchtenden Gründen nie präsent.

Man könnte sogar noch einen Schritt weiter gehen und zu dem Schluss kommen, dass etwaige Pluspunkte der allgemeinen Wehrpflicht aus der gesellschaftspolitischen Perspektive nicht nur überschätzt werden, sondern dass die Nachteile dieser Wehrform alle Vorteile in der Summe sogar überwiegen. Denn in ein der Zeit angemessenes Soldatenbild, in dem etwa den Kategorien "Motivation«, "Anreize« und »Eigenverantwortung « eine weit größere Bedeutung als bisher zuzumessen ist und damit dem Leitbild des »Staatsbürgers in Uniform « sehr viel näher kommt, fügt sich die bisher praktizierte Zwangsrekrutierung schwerlich ein. Auch in dieser Hinsicht erweist sich die Wehrpflicht also als Modernisierungshindernis bei der Weiterentwicklung der Inneren Führung, d.h. der Etablierung demokratischer Werte und Normen in der Armee. So gesehen könnte man sogar sagen, dass zwischen der Demokratisierung von Streitkräften und der Wehrpflicht eben doch ein Zusammenhang besteht - allerdings eher ein konfliktärer.

Kurz und gut: Auch dieses relativ gewichtigste Argument für die Beibehaltung der Wehrpflicht löst sich bei objektiver Prüfung in nichts auf. Als Schreckensszenario taugt es jedenfalls nicht. Wer dennoch in diesem Zusammenhang von der Gefahr einer "Söldnermentalität « spricht, diffamiert im Grunde nur die 200.000 Berufs- und Zeitsoldaten und -soldatinnen, die gegenwärtig in der Bundeswehr ihren Dienst leisten. Eine Wehrform, die mit derart verzweifelten »Begründungen« verteidigt werden muss, ist in Wirklichkeit schon tot.

Diese Einschätzung überwiegt offensichtlich auch in der breiten Öffentlichkeit, insbesondere bei den Jugendlichen als den direkt Betroffenen. Im aktuellen Jahresbericht der Jugendoffiziere, die als wohl wichtigste Träger der Informationsarbeit der Bundeswehr zweifellos über die intensivsten Kontakte mit der genannten Personengruppe verfügen, wird die Situation folgendermaßen beschrieben:

„Die Akzeptanz der allgemeinen Wehrpflicht ist unter Jugendlichen fast gänzlich geschwunden. Auf der allgemeinen Wehrpflicht basierende Streitkräfte werden als Auslaufmodelle gesehen, die den modernen Herausforderungen nicht mehr gewachsen sind. So ist es für die junge Generation auch nur noch eine Frage der Zeit, bis sich auch die Bundesrepublik Deutschland für eine Freiwilligenarmee entscheidet. Die widersprüchlichen Signale aus Politik und Gesellschaft zu diesem Thema werden als Indiz gewertet, dass die allgemeine Wehrpflicht über kurz oder lang fallen wird, zumal nach der Entscheidung, die Einberufung zu ändern, das

3 Kommission »Europäische Sicherheit und Zukunft der Bundeswehr « am IFSH, Demokratie hört nicht am Kasernentor auf. Die Innere Führung in der Bundeswehr muss strukturell verbessert werden, in: Vierteljahresschrift für Sicherheit und Frieden (S+F) 1/2004, S. 45-47. Bald, Detlef/Prüfert, Andreas (Hrsg.), Innere Führung. Ein Plädoyer für eine zweite Militärreform, Baden-Baden 2002.
Gefühl der Wehrgerechtigkeit so gut wie nicht mehr zu vermitteln ist. ${ }^{4}$

\section{III.}

Triftige Gründe für die Beibehaltung der allgemeinen Wehrpflicht in Deutschland gibt es nicht mehr. Nach vielen Jahren Debatte, bei der nun wirklich - auch auf der gesellschaftspolitischen Ebene - jedes mögliche Argument aufgegriffen und hin und her gewendet wurde, ist nun die Zeit überreif für eine politische Entscheidung zur Änderung der Wehrform.

Um es daher auf einen Nenner zu bringen: Die Wehrpflicht geht, die Freiwilligenarmee kommt - die Notwendigkeit einer permanenten Weiterentwicklung der Inneren Führung bleibt.
4 Bundesministerium der Verteidigung, Jahresbericht der Jugendoffiziere der Bundeswehr, Ausgabe 2003, Berlin 21.5.2004, S. 9. 